\title{
Polar Codes: Characterization of Exponent, Bounds, and Constructions
}

\author{
Satish Babu Korada, Eren Şaşoğlu and Rüdiger Urbanke
}

\begin{abstract}
Polar codes were recently introduced by Arikan. They achieve the capacity of arbitrary symmetric binary-input discrete memoryless channels under a low complexity successive cancellation decoding strategy. The original polar code construction is closely related to the recursive construction of ReedMuller codes and is based on the $2 \times 2$ matrix $\left[\begin{array}{ll}1 & 0 \\ 1 & 1\end{array}\right]$. It was shown by Arkan and Telatar that this construction achieves an error exponent of $\frac{1}{2}$, i.e., that for sufficiently large blocklengths the error probability decays exponentially in the square root of the length. It was already mentioned by Arikan that in principle larger matrices can be used to construct polar codes. A fundamental question then is to see whether there exist matrices with exponent exceeding $\frac{1}{2}$. We first show that any $\ell \times \ell$ matrix none of whose column permutations is upper triangular polarizes symmetric channels. We then characterize the exponent of a given square matrix and derive upper and lower bounds on achievable exponents. Using these bounds we show that there are no matrices of size less than 15 with exponents exceeding $\frac{1}{2}$. Further, we give a general construction based on BCH codes which for large $n$ achieves exponents arbitrarily close to 1 and which exceeds $\frac{1}{2}$ for size 16 .
\end{abstract}

\section{INTRODUCTION}

Polar codes, introduced by Arikan in [1], are the first provably capacity achieving codes for arbitrary symmetric binary-input discrete memoryless channels (B-DMC) with low encoding and decoding complexity. The polar code construction is based on the following observation: Let

$$
G_{2}=\left[\begin{array}{ll}
1 & 0 \\
1 & 1
\end{array}\right]
$$

Apply the transform $G_{2}^{\otimes n}$ (where “ $\otimes n$ " denotes the $n^{t h}$ Kronecker power) to a block of $N=2^{n}$ bits and transmit the output through independent copies of a B-DMC $W$ (see Figure 1). As $n$ grows large, the channels seen by individual bits (suitably defined in [1]) start polarizing: they approach either a noiseless channel or a pure-noise channel, where the fraction of channels becoming noiseless is close to the symmetric mutual information $I(W)$.

It was conjectured in [1] that polarization is a general phenomenon, and is not restricted to the particular transformation $G_{2}^{\otimes n}$. In this paper we first give a partial affirmation to this conjecture. In particular, we consider transformations of the form $G^{\otimes n}$ where $G$ is an $\ell \times \ell$ matrix for $\ell \geq 3$ and provide necessary and sufficient conditions for such $G$ s to polarize symmetric B-DMCs.

For the matrix $G_{2}$ it was shown by Arrkan and Telatar [2] that the block error probability for polar coding and successive cancellation decoding is $O\left(2^{-2^{n \beta}}\right)$ for any fixed $\beta<\frac{1}{2}$, where $2^{n}$ is the blocklength. In this case we say that $G_{2}$ has exponent

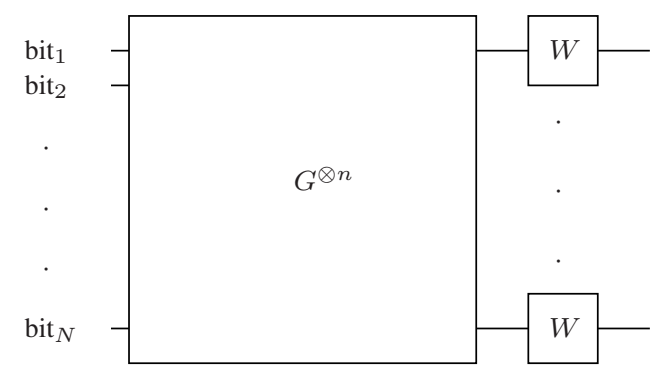

Fig. 1. The transform $G^{\otimes n}$ is applied and the resulting vector is transmitted through the channel $W$.

$\frac{1}{2}$. We show that this exponent can be improved by considering larger matrices. In fact, the exponent can be made arbitrarily close to 1 by increasing the size of the matrix $G$.

Finally, we give an explicit construction of a family of matrices, derived from $\mathrm{BCH}$ codes, with exponent approaching 1 for large $\ell$. This construction results in a matrix whose exponent exceeds $\frac{1}{2}$ for $\ell=16$.

\section{PRELiminaries}

In this paper we deal exclusively with symmetric channels:

Definition 1: A binary-input discrete memoryless channel (B-DMC) $W:\{0,1\} \rightarrow \mathcal{Y}$ is said to be symmetric if there exists a permutation $\pi: \mathcal{Y} \rightarrow \mathcal{Y}$ such that $\pi=\pi^{-1}$ and $W(y \mid 0)=W(\pi(y) \mid 1)$ for all $y \in \mathcal{Y}$.

Let $W:\{0,1\} \rightarrow \mathcal{Y}$ be a symmetric binary-input discrete memoryless channel (B-DMC). Let $I(W) \in[0,1]$ denote the mutual information between the input and output of $W$ with uniform distribution on the inputs. Also, let $Z(W) \in[0,1]$ denote the Bhattacharyya parameter of $W$, i.e., $Z(W)=$ $\sum_{y \in \mathcal{Y}} \sqrt{W(y \mid 0) W(y \mid 1)}$.

Fix an $\ell \geq 3$ and an $\ell \times \ell$ invertible matrix $G$ with entries in $\{0,1\}$. Consider a random $\ell$-vector $U_{1}^{\ell}$ that is uniformly distributed over $\{0,1\}^{\ell}$. Let $X_{1}^{\ell}=U_{1}^{\ell} G$, where the multiplication is performed over $\operatorname{GF}(2)$. Also, let $Y_{1}^{\ell}$ be the output of $\ell$ uses of $W$ with the input $X_{1}^{\ell}$. The channel between $U_{1}^{\ell}$ and $Y_{1}^{\ell}$ is defined by the transition probabilities

$$
W_{\ell}\left(y_{1}^{\ell} \mid u_{1}^{\ell}\right) \triangleq \prod_{i=1}^{\ell} W\left(y_{i} \mid x_{i}\right)=\prod_{i=1}^{\ell} W\left(y_{i} \mid\left(u_{1}^{\ell} G\right)_{i}\right) .
$$

Define $W^{(i)}:\{0,1\} \rightarrow \mathcal{Y}^{\ell} \times\{0,1\}^{i-1}$ as the channel with input $u_{i}$, output $\left(y_{1}^{\ell}, u_{1}^{i-1}\right)$ and transition probabilities

$$
W^{(i)}\left(y_{1}^{\ell}, u_{1}^{i-1} \mid u_{i}\right)=\frac{1}{2^{\ell-1}} \sum_{u_{i+1}^{\ell}} W_{\ell}\left(y_{1}^{\ell} \mid u_{1}^{\ell}\right),
$$


and let $Z^{(i)}$ denote its Bhattacharyya parameter, i.e.,

$$
Z^{(i)}=\sum_{y_{1}^{\ell}, u_{1}^{i-1}} \sqrt{W^{(i)}\left(y_{1}^{\ell}, u_{1}^{i-1} \mid 0\right) W^{(i)}\left(y_{1}^{\ell}, u_{1}^{i-1} \mid 1\right)} .
$$

For $k \geq 1$ let $W^{k}:\{0,1\} \rightarrow \mathcal{Y}^{k}$ denote the B-DMC with transition probabilities

$$
W^{k}\left(y_{1}^{k} \mid x\right)=\prod_{j=1}^{k} W\left(y_{j} \mid x\right) .
$$

Also let $\tilde{W}^{(i)}:\{0,1\} \rightarrow \mathcal{Y}^{\ell}$ denote the B-DMC with transition probabilities

$$
\tilde{W}^{(i)}\left(y_{1}^{\ell} \mid u_{i}\right)=\frac{1}{2^{\ell-i}} \sum_{u_{i+1}^{\ell}} W_{\ell}\left(y_{1}^{\ell} \mid 0_{1}^{i-1}, u_{i}^{\ell}\right) .
$$

Observation 2: Since $W$ is symmetric, the channels $W^{(i)}$ and $\tilde{W}^{(i)}$ are equivalent in the sense that for any fixed $u_{1}^{i-1}$ there exists a permutation $\pi_{u_{1}^{i-1}}: \mathcal{Y}^{\ell} \rightarrow \mathcal{Y}^{\ell}$ such that

$$
W^{(i)}\left(y_{1}^{\ell}, u_{1}^{i-1} \mid u_{i}\right)=\frac{1}{2^{i-1}} \tilde{W}^{(i)}\left(\pi_{u_{1}^{i-1}}\left(y_{1}^{\ell}\right) \mid u_{i}\right) .
$$

Finally, let $I^{(i)}$ denote the mutual information between the input and output of channel $W^{(i)}$. Since $G$ is invertible, it is easy to check that

$$
\sum_{i=1}^{\ell} I^{(i)}=\ell I(W)
$$

We will use $C$ to denote a linear code and $\operatorname{dmin}(C)$ to denote its minimum distance. We let $\left\langle g_{1}, \ldots, g_{k}\right\rangle$ denote the linear code generated by the vectors $g_{1}, \ldots, g_{k}$. We let $d_{H}(a, b)$ denote the Hamming distance between binary vectors $a$ and $b$. We also let $d_{H}(a, \mathrm{C})$ denote the minimum distance between a vector $a$ and a code C, i.e., $d_{H}(a, \mathrm{C})=\min _{c \in \mathrm{C}} d_{H}(a, c)$.

\section{POLARIZATION}

We say that $G$ is a polarizing matrix if there exists an $i \in$ $\{1, \ldots, \ell\}$ for which

$$
\tilde{W}^{(i)}\left(y_{1}^{\ell} \mid u_{i}\right)=Q\left(y_{A^{c}}\right) \prod_{j \in A} W\left(y_{j} \mid u_{i}\right)
$$

for some and $A \subseteq\{1, \ldots, \ell\}$ with $|A|=k, k \geq 2$, and a probability distribution $Q: \mathcal{Y}^{\left|A^{c}\right|} \rightarrow[0,1]$.

In words, a matrix $G$ is polarizing if there exists a bit which "sees" a channel whose $k$ outputs are equivalent to those of $k$ independent realizations of the underlying channel, whereas the remaining $\ell-k$ outputs are independent of the input to the channel. The reason to call such a $G$ "polarizing" is that, as we will see shortly, a repeated application of such a transformation polarizes the underlying channel.

Recall that by assumption $W$ is symmetric. Hence, by Observation 2, equation (5) implies

$$
W^{(i)}\left(y_{1}^{\ell}, u_{1}^{i-1} \mid u_{i}\right)=\frac{Q\left(y_{A^{c}}\right)}{2^{i-1}} \prod_{j \in A} W\left(\left(\pi_{u_{1}^{i-1}}\left(y_{1}^{\ell}\right)\right)_{j} \mid u_{i}\right),
$$

an equivalence we will denote by $W^{(i)} \equiv W^{k}$. Note that $W^{(i)} \equiv W^{k}$ implies $I^{(i)}=I\left(W^{k}\right)$ and $Z^{(i)}=Z\left(W^{k}\right)$.
We start by claiming that any invertible $\{0,1\}$ matrix $G$ can be written as a (real) sum $G=P+P^{\prime}$, where $P$ is a permutation matrix, and $P^{\prime}$ is a $\{0,1\}$ matrix. To see this, consider a bipartite graph on $2 \ell$ nodes. The $\ell$ left nodes correspond to the rows of the matrix and the $\ell$ right nodes correspond to the columns of the matrix. Connect left node $i$ to right node $j$ if $G_{i j}=1$. The invertibility of $G$ implies that for every subset of rows $\mathcal{R}$ the number of columns which contain non-zero elements in these rows is at least $|\mathcal{R}|$. By Hall's Theorem [3, Theorem 16.4.] this guarantees that there is a matching between the left and the right nodes of the graph and this matching represents a permutation. Therefore, for any invertible matrix $G$, there exists a column permutation so that all diagonal elements of the permuted matrix are 1 . Note that the transition probabilities defining $W^{(i)}$ are invariant (up to a permutation of the outputs $y_{1}^{\ell}$ ) under column permutations on $G$. Therefore, for the remainder of this section, and without loss of generality, we assume that $G$ has 1 s on its diagonal.

The following lemma gives necessary and sufficient conditions for (5) to be satisfied.

Lemma 3 (Channel Transformation for Polarizing Matrices): Let $W$ be a symmetric B-DMC.

(i) If $G$ is not upper triangular, then there exists an $i$ for which $W^{(i)} \equiv W^{k}$ for some $k \geq 2$.

(ii) If $G$ is upper triangular, then $W^{(i)} \equiv W$ for all $1 \leq i \leq \ell$. Proof: Let the number of $1 \mathrm{~s}$ in the last row of $G$ be $k$. Clearly $W^{(\ell)} \equiv W^{k}$. If $k \geq 2$ then $G$ is not upper triangular and the first claim of the lemma holds. If $k=1$ then

$$
G_{l k}=0, \text { for all } 1 \leq k<\ell .
$$

One can then write

$$
\begin{aligned}
& W^{(\ell-i)}\left(y_{1}^{\ell}, u_{1}^{\ell-i-1} \mid u_{\ell-i}\right) \\
& =\frac{1}{2^{\ell-1}} \sum_{u_{\ell-i+1}^{\ell}} W_{\ell}\left(y_{1}^{\ell} \mid u_{1}^{\ell}\right) \\
& =\frac{1}{2^{\ell-1}} \sum_{u_{\ell-i+1}^{\ell-1}, u_{\ell}} \operatorname{Pr}\left[Y_{1}^{\ell-1}=y_{1}^{\ell-1} \mid U_{1}^{\ell}=u_{1}^{\ell}\right] \\
& \cdot \operatorname{Pr}\left[Y_{\ell}=y_{\ell} \mid Y_{1}^{\ell-1}=y_{1}^{\ell-1}, U_{1}^{\ell}=u_{1}^{\ell}\right] \\
& \begin{array}{c}
\stackrel{(7)}{=} \frac{1}{2^{\ell-1}} \sum_{u_{\ell-i+1}^{\ell-1}, u_{\ell}} W_{\ell-1}\left(y_{1}^{\ell-1} \mid u_{1}^{\ell-1}\right) \\
\quad \cdot \operatorname{Pr}\left[Y_{\ell}=y_{\ell} \mid Y_{1}^{\ell-1}=y_{1}^{\ell-1}, U_{1}^{\ell}=u_{1}^{\ell}\right] \\
=\frac{1}{2^{\ell-1}} \sum_{u_{\ell-i+1}^{\ell-1}} W_{\ell-1}\left(y_{1}^{\ell-1} \mid u_{1}^{\ell-1}\right) \\
\quad \sum_{u_{\ell}} \operatorname{Pr}\left[Y_{\ell}=y_{\ell} \mid Y_{1}^{\ell-1}=y_{1}^{\ell-1}, U_{1}^{\ell}=u_{1}^{\ell}\right] \\
\left.=\frac{1}{2^{\ell-1}}\left[W\left(y_{\ell} \mid 0\right)+W_{(} \mid 1\right)\right] \sum_{u_{\ell-i+1}^{\ell-1}} W_{\ell-1}\left(y_{1}^{\ell-1} \mid u_{1}^{\ell-1}\right) .
\end{array}
\end{aligned}
$$

Therefore, $Y_{\ell}$ is independent of the inputs to the channels $W^{(\ell-i)}$ for $i=1, \ldots, \ell-1$. This is equivalent to saying that channels $W^{(1)}, \ldots, W^{(\ell-1)}$ are defined by the matrix $G^{(\ell-1)}$, where we define $G^{(\ell-i)}$ as the $(\ell-i) \times(\ell-i)$ matrix obtained from $G$ by removing its last $i$ rows and columns. Applying 
the same argument to $G^{(\ell-1)}$ and repeating, we see that if $G$ is upper triangular, then we have $W^{(i)} \equiv W$ for all $i$. On the other hand, if $G$ is not upper triangular, then there exists an $i$ for which $G^{(\ell-i)}$ has at least two $1 \mathrm{~s}$ in the last row. This in turn implies that $W^{(i)} \equiv W^{k}$ for some $k \geq 2$.

Consider the recursive channel combining operation given in [1], using a transformation $G$. Recall that $n$ recursions of this construction is equivalent to applying the transformation $A_{n} G^{\otimes n}$ to $U_{1}^{\ell^{n}}$ where, $A_{n}:\left\{1, \ldots, \ell^{n}\right\} \rightarrow\left\{1, \ldots, \ell^{n}\right\}$ is a permutation defined analogously to the bit-reversal operation in [1].

Theorem 4 (Polarization of Symmetric B-DMCs): Given a symmetric B-DMC $W$ and an $\ell \times \ell$ transformation $G$, consider the channels $W^{(i)}, i=\left\{1, \ldots, \ell^{n}\right\}$, defined by the transformation $A_{n} G^{\otimes n}$.

(i) If $G$ is polarizing, then for any $\delta>0$

$$
\begin{aligned}
& \lim _{n \rightarrow \infty} \frac{\left|\left\{i \in\left\{1, \ldots, \ell^{n}\right\}: I\left(W^{(i)}\right) \in(\delta, 1-\delta)\right\}\right|}{\ell^{n}}=0, \\
& \lim _{n \rightarrow \infty} \frac{\left|\left\{i \in\left\{1, \ldots, \ell^{n}\right\}: Z\left(W^{(i)}\right) \in(\delta, 1-\delta)\right\}\right|}{\ell^{n}}=0 .
\end{aligned}
$$

(ii) If $G$ is not polarizing, then for all $n$ and $i \in\left\{1, \ldots, \ell^{n}\right\}$

$$
I\left(W^{(i)}\right)=I(W), \quad Z\left(W^{(i)}\right)=Z(W) .
$$

In [1, Section 6], Arıkan proves part (i) of Theorem 4 for $G=G_{2}$. His proof involves defining a random variable $W_{n}$ that is uniformly distributed over the set $\left\{W^{(i)}\right\}_{i=1}^{\ell^{n}}$ (where $\ell=2$ for the case $G=G_{2}$ ), which implies

$$
\operatorname{Pr}\left[I\left(W_{n}\right) \in(a, b)\right]=\frac{\left|\left\{i \in\left\{1, \ldots, \ell^{n}\right\}: I\left(W^{(i)}\right) \in(a, b)\right\}\right|}{\ell^{n}},
$$

$\operatorname{Pr}\left[Z\left(W_{n}\right) \in(a, b)\right]=\frac{\left|\left\{i \in\left\{1, \ldots, \ell^{n}\right\}: Z\left(W^{(i)}\right) \in(a, b)\right\}\right|}{\ell^{n}}$

Following Arıkan, we define the random variable $W_{n} \in$ $\left\{W^{(i)}\right\}_{i=1}^{\ell^{n}}$ for our purpose through a tree process $\left\{W_{n} ; n \geq\right.$ $0\}$ with

$$
\begin{aligned}
W_{0} & =W, \\
W_{n+1} & =W_{n}^{\left(B_{n+1}\right)},
\end{aligned}
$$

where $\left\{B_{n} ; n \geq 1\right\}$ is a sequence of i.i.d. random variables defined on a probability space $(\Omega, \mathcal{F}, \mu)$, and where $B_{n}$ is uniformly distributed over the set $\{1, \ldots, \ell\}$. Defining $\mathcal{F}_{0}=$ $\{\emptyset, \Omega\}$ and $\mathcal{F}_{n}=\sigma\left(B_{1}, \ldots, B_{n}\right)$ for $n \geq 1$, we augment the above process by the processes $\left\{I_{n} ; n \geq 0\right\}:=\left\{I\left(W_{n}\right) ; n \geq\right.$ $0\}$ and $\left\{Z_{n} ; n \geq 0\right\}:=\left\{Z\left(W_{n}\right) ; n \geq 0\right\}$. It is easy to verify that these processes satisfy (10) and (11).

Observation 5: $\left\{\left(I_{n}, \mathcal{F}_{n}\right)\right\}$ is a bounded martingale and therefore converges w.p. 1 and in $\mathcal{L}^{1}$ to a random variable $I_{\infty}$

Lemma $6\left(I_{\infty}\right)$ : If $G$ is polarizing, then

$$
I_{\infty}= \begin{cases}1 & \text { w.p. } I(W), \\ 0 & \text { w.p. } 1-I(W) .\end{cases}
$$

Proof: For any polarizing transformation $G$, Lemma 3 implies that there exists an $i \in\{1, \ldots, \ell\}$ and $k \geq 2$ for which

$$
I^{(i)}=I\left(W^{k}\right) \text {. }
$$

This implies that for the tree process defined above, we have

$$
I_{n+1}=I\left(W_{n}^{k}\right) \text { with probability at least } \frac{1}{\ell},
$$

for some $k \geq 2$. Moreover by the convergence in $\mathcal{L}^{1}$ of $I_{n}$, we have $\mathbb{E}\left[\left|\bar{I}_{n+1}-I_{n}\right|\right] \stackrel{n \rightarrow \infty}{\longrightarrow} 0$. This in turn implies

$$
\mathbb{E}\left[\left|I_{n+1}-I_{n}\right|\right] \geq \frac{1}{\ell} \mathbb{E}\left[\left(I\left(W_{n}^{k}\right)-I\left(W_{n}\right)\right] \rightarrow 0 .\right.
$$

It is shown in Lemma 33 in the Appendix that for any symmetric B-DMC $W_{n}$, if $I\left(W_{n}\right) \in(\delta, 1-\delta)$ for some $\delta>0$, then there exists an $\eta(\delta)>0$ such that $I\left(W_{n}^{k}\right)-I\left(W_{n}\right)>$ $\eta(\delta)$. Therefore, convergence in (13) implies $I_{\infty} \in\{0,1\}$ w.p. 1 . The claim on the probability distribution of $I_{\infty}$ follows from the fact that $\left\{I_{n}\right\}$ is a martingale, i.e., $\mathbb{E}\left[I_{\infty}\right]=\mathbb{E}\left[I_{0}\right]=I(W)$.

Proof of Theorem 4: Note that for any $n$ the fraction in (8) is equal to $\operatorname{Pr}\left[I_{n} \in(\delta, 1-\delta)\right]$. Combined with Lemma 6, this implies (8).

For any B-DMC $Q, I(Q)$ and $Z(Q)$ satisfy [1]

$$
\begin{aligned}
I(Q)^{2}+Z(Q)^{2} & \leq 1, \\
I(Q)+Z(Q) & \geq 1 .
\end{aligned}
$$

When $I(Q)$ takes on the value 0 or 1 , these two inequalities imply that $Z(Q)$ takes on the value 1 or 0 , respectively. From Lemma 6 we know that $\left\{I_{n}\right\}$ converges to $I_{\infty}$ w.p. 1 and $I_{\infty} \in\{0,1\}$. This implies that $\left\{Z_{n}\right\}$ converges w.p. 1 to a random variable $Z_{\infty}$ and

$$
Z_{\infty}= \begin{cases}0 & \text { w.p. } I(W), \\ 1 & \text { w.p. } 1-I(W) .\end{cases}
$$

This proves the first part of the theorem. The second part follows from Lemma 3, (ii).

Remark 7: Arikan's proof for part (i) of Theorem 4 with $G=G_{2}$ proceeds by first showing the convergence of $\left\{Z_{n}\right\}$, instead of $\left\{I_{n}\right\}$. This is accomplished by showing that for the matrix $G_{2}$ the resulting process $\left\{Z_{n}\right\}$ is a submartingale. Such a property is in general difficult to prove for arbitrary $G$. On the other hand, the process $\left\{I_{n}\right\}$ is a martingale for any invertible matrix $G$, which is sufficient to ensure convergence.

Theorem 4 guarantees that repeated application of a polarizing matrix $G$ polarizes the underlying channel $W$, i.e., the resulting channels $W^{(i)}, i \in\left\{1, \ldots, \ell^{n}\right\}$, tend towards either a noiseless or a completely noisy channel. Lemma 6 ensures that the fraction of noiseless channels is indeed $I(W)$. This suggests to use the noiseless channels for transmitting information while transmitting no information over the noisy channels [1]. Let $\mathcal{A} \subset\left\{1, \ldots, \ell^{n}\right\}$ denote the set of channels $W^{(i)}$ used for transmitting the information bits. Since $Z^{(i)}$ upper bounds the error probability of decoding bit $U_{i}$ with the knowledge of $U_{1}^{i-1}$, the block error probability of such a transmission scheme under successive cancellation decoder can be upper bounded as [1]

$$
P_{B} \leq \sum_{i \in \mathcal{A}} Z^{(i)} .
$$


Further, the block error probability can also be lower bounded in terms of the $Z^{(i)} \mathrm{s}$ : Consider a symmetric B-DMC with Bhattacharyya parameter $Z$, and let $P_{e}$ denote the bit error probability of uncoded transmission over this channel. It is known that

$$
P_{e} \geq \frac{1}{2}\left(1-\sqrt{1-Z^{2}}\right) .
$$

A proof of this fact is provided in the Appendix. Under successive cancellation decoding, the block error probability is lower bounded by each of the bit error probabilities over the channels $W^{(i)}$. Therefore the former quantity can be lower bounded by

$$
P_{B} \geq \max _{i \in \mathcal{A}} \frac{1}{2}\left(1-\sqrt{1-\left(Z^{(i)}\right)^{2}}\right) .
$$

Both the above upper and lower bounds to the block error probability look somewhat loose at a first look. However, as we shall see later, these bounds are sufficiently tight for our purposes. Therefore, it suffices to analyze the behavior of the $Z^{(i)} \mathrm{s}$.

\section{RATE OF POLARIZATION}

For the matrix $G_{2}$ Arrkan shows that, combined with successive cancellation decoding, these codes achieve a vanishing block error probability for any rate strictly less than $I(W)$. Moreover, it is shown in [2] that when $Z_{n}$ approaches 0 it does so at a sufficiently fast rate:

Theorem 8 ([2]): Given a B-DMC $W$, the matrix $G_{2}$ and any $\beta<\frac{1}{2}$,

$$
\lim _{n \rightarrow \infty} \operatorname{Pr}\left[Z_{n} \leq 2^{-2^{n \beta}}\right]=I(W) .
$$

A similar result for arbitrary $G$ is given in the following theorem.

Theorem 9 (Universal Bound on Rate of Polarization): Given a symmetric B-DMC $W$, an $\ell \times \ell$ polarizing matrix $G$, and any $\beta<\frac{\log _{\ell} 2}{\ell}$,

$$
\lim _{n \rightarrow \infty} \operatorname{Pr}\left[Z_{n} \leq 2^{-\ell^{n \beta}}\right]=I(W) .
$$

Proof Idea: For any polarizing matrix it can be shown that $Z_{n+1} \leq \ell Z_{n}$ with probability 1 and that $Z_{n+1} \leq Z_{n}^{2}$ with probability at least $1 / \ell$. The proof then follows by adapting the proof of [2, Theorem 3].

The above estimation of the probability is universal and is independent of the exact structure of $G$. We are now interested in a more precise estimate of this probability. The results in this section are the natural generalization of those in [2].

Definition 10 (Rate of Polarization): For any B-DMC $W$ with $0<I(W)<1$, we will say that an $\ell \times \ell$ matrix $G$ has rate of polarization $\mathrm{E}(G)$ if

(i) For any fixed $\beta<\mathrm{E}(G)$,

$$
\liminf _{n \rightarrow \infty} \operatorname{Pr}\left[Z_{n} \leq 2^{-\ell^{n \beta}}\right]=I(W) .
$$

(ii) For any fixed $\beta>\mathrm{E}(G)$,

$$
\liminf _{n \rightarrow \infty} \operatorname{Pr}\left[Z_{n} \geq 2^{-\ell^{n \beta}}\right]=1 .
$$

For convenience, in the rest of the paper we refer to $\mathrm{E}(G)$ as the exponent of the matrix $G$.
The definition of exponent provides a meaningful performance measure of polar codes under successive cancellation decoding. This can be seen as follows: Consider a matrix $G$ with exponent $\mathrm{E}(G)$. Fix $0<R<I(W)$ and $\beta<\mathrm{E}(G)$. Definition 10 (i) implies that for $n$ sufficiently large there exists a set $\mathcal{A}$ of size $\ell^{n} R$ such that $\sum_{i \in \mathcal{A}} Z^{(i)} \leq 2^{-\ell^{n \beta}}$. Using set $\mathcal{A}$ as the set of information bits, the block error probability under successive cancellation decoding $P_{B}$ can be bounded using (14) as

$$
P_{B} \leq 2^{-\ell^{n \beta}} .
$$

Conversely, consider $R>0$ and $\beta>\mathrm{E}(G)$. Definition 10 (ii) implies that for $n$ sufficiently large, any set $\mathcal{A}$ of size $\ell^{n} R$ will satisfy $\max _{i \in \mathcal{A}} Z^{(i)}>2^{-\ell^{n \beta}}$. Using (15) the block error probability can be lower bounded as

$$
P_{B} \geq 2^{-\ell^{n \beta}} \text {. }
$$

It turns out, and it will be shown later, that the exponent is independent of the channel $W$. Indeed, we will show in Theorem 14 that the exponent $\mathrm{E}(G)$ can be expressed as a function of the partial distances of $G$.

Definition 11 (Partial Distances): Given an $\ell \times \ell$ matrix $G=\left[g_{1}^{T}, \ldots, g_{\ell}^{T}\right]^{T}$, we define the partial distances $D_{i}$, $i=1, \ldots, \ell$ as

$$
\begin{aligned}
& D_{i} \triangleq d_{H}\left(g_{i},\left\langle g_{i+1}, \ldots, g_{\ell}\right\rangle\right), \quad i=1, \ldots, \ell-1, \\
& D_{\ell} \triangleq d_{H}\left(g_{\ell}, 0\right) .
\end{aligned}
$$

Example 12: The partial distances of the matrix

$$
F=\left[\begin{array}{lll}
1 & 0 & 0 \\
1 & 0 & 1 \\
1 & 1 & 1
\end{array}\right]
$$

are $D_{1}=1, D_{2}=1, D_{3}=3$.

In order to establish the relationship between $\mathrm{E}(G)$ and the partial distances of $G$ we consider the Bhattacharyya parameters $Z^{(i)}$ of the channels $W^{(i)}$. These parameters depend on $G$ as well as on $W$. The exact relationship with respect to $W$ is difficult to compute in general. However, there are sufficiently tight upper and lower bounds on the $Z^{(i)} \mathrm{s}$ in terms of $Z(W)$, the Battacharyya parameter of $W$.

Lemma 13 (Bhattacharyya Parameter and Partial Distance): For any symmetric B-DMC $W$ and any $\ell \times \ell$ matrix $G$ with partial distances $\left\{D_{i}\right\}_{i=1}^{\ell}$

$$
Z(W)^{D_{i}} \leq Z^{(i)} \leq 2^{\ell-i} Z(W)^{D_{i}}
$$

Proof: To prove the upper bound we write

$$
\begin{aligned}
Z^{(i)} & =\sum_{y_{1}^{\ell}, u_{1}^{i-1}} \sqrt{W^{(i)}\left(y_{1}^{\ell}, u_{1}^{i-1} \mid 0\right) W^{(i)}\left(y_{1}^{\ell}, u_{1}^{i-1} \mid 1\right)} \\
& \stackrel{(3)}{=} \frac{1}{2^{\ell-1}} \sum_{y_{1}^{\ell}, u_{1}^{i-1}} \\
& \sqrt{\sum_{v_{i+1}^{\ell}, w_{i+1}^{\ell}} W_{\ell}\left(y_{1}^{\ell} \mid u_{1}^{i-1}, 0, v_{i+1}^{\ell}\right) W_{\ell}\left(y_{1}^{\ell} \mid u_{1}^{i-1}, 1, w_{i+1}^{\ell}\right)} \\
\leq & \frac{1}{2^{\ell-1}} \sum_{y_{1}^{\ell}, u_{1}^{i-1}} \sum_{v_{i+1}^{\ell}, w_{i+1}^{\ell}} \sqrt{W_{\ell}\left(y_{1}^{\ell} \mid u_{1}^{i-1}, 0, v_{i+1}^{\ell}\right)}
\end{aligned}
$$




$$
\cdot \sqrt{W_{\ell}\left(y_{1}^{\ell} \mid u_{1}^{i-1}, 1, w_{i+1}^{\ell}\right)} .
$$

Let $c_{0}=\left(u_{1}^{i-1}, 0, v_{i+1}^{\ell}\right) G$ and $c_{1}=\left(u_{1}^{i-1}, 1, w_{i+1}^{\ell}\right) G$. Let $S_{0}\left(S_{1}\right)$ be the set of indices where both $c_{0}$ and $c_{1}$ are equal to 0(1). Let $S^{c}$ be the complement of $S_{0} \cup S_{1}$. We have

$$
\left|S^{c}\right|=d_{H}\left(c_{0}, c_{1}\right) \geq D_{i} .
$$

Now, (17) can be rewritten as

$$
\begin{aligned}
Z^{(i)} & \leq \frac{1}{2^{\ell-1}} \sum_{v_{i+1}^{\ell}, w_{i+1}^{\ell}} \sum_{y_{1}^{\ell}, u_{1}^{i-1}} \prod_{j \in S_{0}} W\left(y_{j} \mid 0\right) \prod_{j \in S_{1}} W\left(y_{j} \mid 1\right) \\
& \cdot \sqrt{\prod_{j \in S^{c}} W\left(y_{j} \mid 0\right) W\left(y_{j} \mid 1\right)} \\
& \leq \frac{1}{2^{\ell-1}} \sum_{v_{i+1}^{\ell}, w_{i+1}^{\ell}, u_{1}^{i-1}} Z^{D_{i}} \\
& =2^{\ell-i} Z^{D_{i}} .
\end{aligned}
$$

For the lower bound on $Z^{(i)}$, first note that by Observation 2, we have $Z\left(W^{(i)}\right)=Z\left(\tilde{W}^{(i)}\right)$. Therefore it suffices to show the claim for the channel $\tilde{W}^{(i)}$. Let $G=\left[g_{1}^{T}, \ldots, g_{\ell}^{T}\right]^{T}$. Then using (2), (3) and (4), $\tilde{W}^{(i)}$ can be written as

$$
\tilde{W}^{(i)}\left(y_{1}^{\ell} \mid u_{i}\right)=\frac{1}{2^{\ell-i}} \sum_{x_{1}^{\ell} \in \mathcal{A}\left(u_{i}\right)} \prod_{k=1}^{\ell} W\left(y_{k} \mid x_{k}\right)
$$

where $x_{1}^{\ell} \in \mathcal{A}\left(u_{i}\right) \subset\{0,1\}^{\ell}$ if and only if for some $u_{i+1}^{\ell} \in$ $\{0,1\}^{\ell-i}$

$$
x_{1}^{\ell}=u_{i} g_{i}+\sum_{j=i+1}^{\ell} u_{j} g_{j} .
$$

Consider the code $\left\langle g_{i+1}, \ldots, g_{\ell}\right\rangle$ and let $\sum_{j=i+1}^{\ell} \alpha_{j} g_{j}$ be a codeword satisfying $d_{H}\left(g_{i}, \sum_{j=i+1}^{\ell} \alpha_{j} g_{j}\right)=D_{i}$. Due to the linearity of the code $\left\langle g_{i+1} \ldots, g_{\ell}\right\rangle$, one can equivalently say that $x_{1}^{\ell} \in \mathcal{A}\left(u_{i}\right)$ if and only if

$$
x_{1}^{\ell}=u_{i}\left(g_{i}+\sum_{j=i+1}^{\ell} \alpha_{j} g_{j}\right)+\sum_{j=i+1}^{\ell} u_{j} g_{j} .
$$

Now let $g_{i}^{\prime}=g_{i}+\sum_{j=i+1}^{\ell} \alpha_{j} g_{j}$ and $G^{\prime}=$ $\left[g_{1}^{T}, \ldots, g_{i-1}^{T}, g_{i}^{\prime T}, g_{i+1}^{T}, \ldots, g_{\ell}^{T}\right]^{T}$. Equations (19) and (20) show that the channels $W^{(i)}$ defined by the matrices $G$ and $G^{\prime}$ are equivalent. Note that $G^{\prime}$ has the property that the Hamming weight of $g_{i}^{\prime}$ is equal to $D_{i}$.

We will now consider a channel $W_{g}^{(i)}$ where a genie provides extra information to the decoder. Since $\tilde{W}^{(i)}$ is degraded with respect to the genie-aided channel $W_{g}^{(i)}$, and since the ordering of the Bhattacharyya parameter is preserved under degradation, it suffices to find a genie-aided channel for which $Z_{g}^{(i)}=Z(W)^{D_{i}}$.

Consider a genie which reveals the bits $u_{i+1}^{\ell}$ to the decoder (Figure 2). With the knowledge of $u_{i+1}^{\ell}$ the decoder's task reduces to finding the value of any of the transmitted bits $x_{j}$ for which $g_{i j}=1$. Since each bit $x_{j}$ goes through an

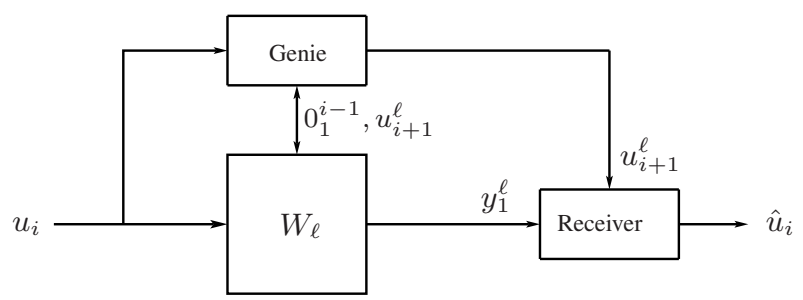

Fig. 2. Genie-aided channel $W_{g}^{(i)}$.

independent copy of $W$, and since the weight of $g_{i}$ is equal to $D_{i}$, the resulting channel $W_{g}^{(i)}$ is equivalent to $D_{i}$ independent copies of $W$. Hence, $Z_{g}^{(i)}=Z(W)^{D_{i}}$.

Lemma 13 shows that the link between $Z^{(i)}$ and $Z(W)$ is given in terms of the partial distances of $G$. This link is sufficiently strong to completely characterize $\mathrm{E}(G)$.

Theorem 14 (Exponent from Partial Distances): For any symmetric B-DMC $W$ and any $\ell \times \ell$ matrix $G$ with partial distances $\left\{D_{i}\right\}_{i=1}^{\ell}$, the rate of polarization $\mathrm{E}(G)$ is given by

$$
\mathrm{E}(G)=\frac{1}{\ell} \sum_{i=1}^{\ell} \log _{\ell} D_{i}
$$

Proof: The proof is similar to that of [2, Theorem 3]. We highlight the main idea and omit the details.

First note that by Lemma 13 we have $Z_{j} \geq Z_{j-1}^{D_{B_{j}}}$. Let $m_{i}=\left|\left\{1 \leq j \leq n: B_{j}=i\right\}\right|$. We then obtain

$$
Z_{n} \geq Z^{\prod_{i} D_{i}^{m_{i}}}=Z^{\ell^{\left(\sum_{i} m_{i} \log _{\ell} D_{i}\right)} .}
$$

The exponent of $Z$ on the right-hand side of (22) can be rewritten as

$$
\ell^{\sum_{i} m_{i} \log _{\ell} D_{i}}=\left(\ell^{n}\right)^{\sum_{i} \frac{m_{i}}{n} \log _{\ell} D_{i}} .
$$

By the law of large numbers, for any $\epsilon>0$,

$$
\left|\frac{m_{i}}{n}-\frac{1}{\ell}\right| \leq \epsilon
$$

with high probability for $n$ sufficiently large. This proves part (ii) of the definition of $\mathrm{E}(G)$, i.e., for any $\beta>\frac{1}{\ell} \sum_{i} \log _{\ell} D_{i}$,

$$
\lim _{n \rightarrow \infty} \operatorname{Pr}\left[Z_{n} \geq 2^{-\ell^{n \beta}}\right]=1 .
$$

The proof for part $(i)$ of the definition follows using similar arguments as above, and by noting that $Z_{j} \leq 2^{\ell-B_{j}} Z_{j-1}^{D_{B_{j}}}$. The constant $2^{\ell-B_{j}}$ can be taken care of using the 'bootstrapping' argument of [2].

Example 15: For the matrix $F$ considered in Example 12, we have

$$
\mathrm{E}(F)=\frac{1}{3}\left(\log _{3} 1+\log _{3} 1+\log _{3} 3\right)=\frac{1}{3} .
$$

\section{BOUNDS ON THE EXPONENT}

For the matrix $G_{2}$, we have $\mathrm{E}\left(G_{2}\right)=\frac{1}{2}$. Note that for the case of $2 \times 2$ matrices, the only polarizing matrix is $G_{2}$. In 
order to address the question of whether the rate of polarization can be improved by considering large matrices, we define

$$
\mathrm{E}_{\ell} \triangleq \max _{G \in\{0,1\}^{\ell \times \ell}} \mathrm{E}(G) .
$$

Theorem 14 facilitates the computation of $\mathrm{E}_{\ell}$ by providing an expression for $\mathrm{E}(G)$ in terms of the partial distances of $G$. Lemmas 16 and 18 below provide further simplification for computing (23).

Lemma 16 (Gilbert-Varshamov Inequality for Linear Codes): Let $\mathrm{C}$ be a binary linear code of length $\ell$ and $\operatorname{dmin}(\mathrm{C})=d_{1}$.

Let $g \in\{0,1\}^{\ell}$ and let $d_{H}(g, \mathrm{C})=d_{2}$. Let $\mathrm{C}^{\prime}$ be the linear code obtained by adding the vector $g$ to $\mathrm{C}$, i.e., $\mathrm{C}^{\prime}=\langle g, \mathrm{C}\rangle$. Then $\operatorname{dmin}\left(\mathrm{C}^{\prime}\right)=\min \left\{d_{1}, d_{2}\right\}$.

Proof: Since $\mathrm{C}^{\prime}$ is a linear code, its codewords are of the form $c+\alpha g$ where $c \in \mathrm{C}, \alpha \in\{0,1\}$. Therefore

$$
\begin{aligned}
\operatorname{dmin}\left(\mathrm{C}^{\prime}\right) & =\min _{c \in \mathrm{C}}\left\{\min \left\{d_{H}(0, c), d_{H}(0, c+g)\right\}\right\} \\
& =\min \left\{\min _{c \in \mathrm{C}}\left\{d_{H}(0, c)\right\}, \min _{c \in \mathrm{C}}\left\{d_{H}(g, c)\right\}\right\} \\
& =\min \left\{d_{1}, d_{2}\right\} .
\end{aligned}
$$

Corollary 17: Given a set of vectors $g_{1}, \ldots, g_{k}$ with partial distances $D_{j}=d_{H}\left(g_{j},\left\langle g_{j+1}, \ldots, g_{k}\right\rangle\right), j=1, \ldots, k$, the minimum distance of the linear code $\left\langle g_{1}, \ldots, g_{k}\right\rangle$ is given by $\min _{j=1}^{\ell}\left\{D_{j}\right\}$.

The maximization problem in (23) is not feasible in practice even for $\ell \geq 10$. The following lemma allows to restrict this maximization to a smaller set of matrices. Even though the maximization problem still remains intractable, by working on this restricted set, we obtain lower and upper bounds on $\mathrm{E}_{\ell}$.

Lemma 18 (Partial Distances Should Decrease): Let $G=$ $\left[g_{1}^{T} \ldots g_{\ell}^{T}\right]^{T}$. Fix $k \in\{1, \ldots, \ell\}$ and let $G^{\prime}=$ $\left[g_{1}^{T} \ldots g_{k+1}^{T} g_{k}^{T} \ldots g_{\ell}^{T}\right]^{T}$ be the matrix obtained from $G$ by swapping $g_{k}$ and $g_{k+1}$. Let $\left\{D_{i}\right\}_{i=1}^{\ell}$ and $\left\{D_{i}^{\prime}\right\}_{i=1}^{\ell}$ denote the partial distances of $G$ and $G^{\prime}$ respectively. If $D_{k}>D_{k+1}$, then

(i) $\mathrm{E}\left(G^{\prime}\right) \geq \mathrm{E}(G)$

(ii) $D_{k+1}^{\prime}>D_{k}^{\prime}$.

Proof: Note first that $D_{i}=D_{i}^{\prime}$ if $i \notin\{k, k+1\}$. Therefore, to prove the first claim, it suffices to show that $D_{k}^{\prime} D_{k+1}^{\prime} \geq D_{k} D_{k+1}$. To that end, write

$$
\begin{aligned}
D_{k}^{\prime} & =d_{H}\left(g_{k+1},\left\langle g_{k}, g_{k+2}, \ldots, g_{\ell}\right\rangle\right), \\
D_{k} & =d_{H}\left(g_{k},\left\langle g_{k+1}, \ldots, g_{\ell}\right\rangle\right), \\
D_{k+1}^{\prime} & =d_{H}\left(g_{k},\left\langle g_{k+2}, \ldots, g_{\ell}\right\rangle\right), \\
D_{k+1} & =d_{H}\left(g_{k+1},\left\langle g_{k+2}, \ldots, g_{\ell}\right\rangle\right),
\end{aligned}
$$

and observe that $D_{k+1}^{\prime} \geq D_{k}$ since $\left\langle g_{k+2}, \ldots, g_{\ell}\right\rangle$ is a subcode of $\left\langle g_{k+1}, \ldots, g_{\ell}\right\rangle$. $D_{k}^{\prime}$ can be computed as

$$
\begin{aligned}
& \min \left\{\min _{c \in\left\langle g_{k+2}, \ldots, g_{\ell}\right\rangle} d_{H}\left(g_{k+1}, c\right), \min _{c \in\left\langle g_{k+2}, \ldots, g_{\ell}\right\rangle} d_{H}\left(g_{k+1}, c+g_{k}\right)\right\} \\
& =\min \left\{D_{k+1}, \min _{c \in\left\langle g_{k+2}, \ldots, g_{\ell}\right\rangle} d_{H}\left(g_{k}, c+g_{k+1}\right)\right\} \\
& =D_{k+1},
\end{aligned}
$$

where the last equality follows from

$$
\begin{aligned}
\min _{c \in\left\langle g_{k+2}, \ldots, g_{\ell}\right\rangle} d_{H}\left(g_{k}, c+g_{k+1}\right) & \geq \min _{c \in\left\langle g_{k+1}, g_{k+2}, \ldots, g_{\ell}\right\rangle} d_{H}\left(g_{k}, c\right) \\
& =D_{k}>D_{k+1} .
\end{aligned}
$$

Therefore, $D_{k}^{\prime} D_{k+1}^{\prime} \geq D_{k} D_{k+1}$, which proves the first claim. The second claim follows from the inequality $D_{k+1}^{\prime} \geq D_{k}>$ $D_{k+1}=D_{k}^{\prime}$.

Corollary 19: In the definition of $\mathrm{E}_{\ell}$ (23), the maximization can be restricted to the matrices $G$ which satisfy $D_{1} \leq D_{2} \leq$ $\ldots \leq D_{\ell}$.

\section{A. Lower Bound}

The following lemma provides a lower bound on $\mathrm{E}_{\ell}$ by using a Gilbert-Varshamov type construction.

Lemma 20 (Gilbert-Varshamov Bound):

$$
\mathrm{E}_{\ell} \geq \frac{1}{\ell} \sum_{i=1}^{\ell} \log _{\ell} \tilde{D}_{i}
$$

where

$$
\tilde{D}_{i}=\max \left\{D: \sum_{j=0}^{D-1}\left(\begin{array}{l}
\ell \\
j
\end{array}\right)<2^{i}\right\} .
$$

Proof: We will construct a matrix $G=\left[g_{1}^{T}, \ldots, g_{\ell}^{T}\right]^{T}$, with partial distances $D_{i}=\tilde{D}_{i}$ : Let $S(c, d)$ denote the set of binary vectors with Hamming distance at most $d$ from $c \in$ $\{0,1\}^{\ell}$, i.e.,

$$
S(c, d)=\left\{x \in\{0,1\}^{\ell}: d_{H}(x, c) \leq d\right\} .
$$

To construct the $i^{\text {th }}$ row of $G$ with partial distance $\tilde{D}_{i}$, we will find a $v \in\{0,1\}^{\ell}$ satisfying $d_{H}\left(v,\left\langle g_{i+1}, \ldots, g_{\ell}\right\rangle\right)=\tilde{D}_{i}$ and set $g_{i}=v$. Such a $v$ satisfies $v \notin S\left(c, \tilde{D}_{i}-1\right)$ for all $c \in\left\langle g_{i+1}, \ldots, g_{\ell}\right\rangle$ and exists if the sets $S\left(c, \tilde{D}_{i}-1\right), c \in$ $\left\langle g_{i+1}, \ldots, g_{\ell}\right\rangle$ do not cover $\{0,1\}^{\ell}$. The latter condition is satisfied if

$$
\begin{aligned}
\left|\cup_{c \in\left\langle g_{i+1}, \ldots, g_{\ell}\right\rangle} S\left(c, \tilde{D}_{i}-1\right)\right| & \leq \sum_{c \in\left\langle g_{i+1}, \ldots, g_{\ell}\right\rangle}\left|S\left(c, \tilde{D}_{i}-1\right)\right| \\
& =2^{\ell-i} \sum_{j=0}^{\tilde{D}_{i}-1}\left(\begin{array}{l}
\ell \\
j
\end{array}\right)<2^{\ell}
\end{aligned}
$$

which is guaranteed by (24).

The solid line in Figure 3 shows the lower bound of Lemma 20 . The bound exceeds $\frac{1}{2}$ for $\ell=85$, suggesting that the exponent can be improved by considering large matrices. In fact, the lower bound tends to 1 when $\ell$ tends to infinity:

Lemma 21 (Exponent 1 is Achievable): $\lim _{\ell \rightarrow \infty} \mathrm{E}_{\ell}=1$.

Proof: Fix $\alpha \in\left(0, \frac{1}{2}\right)$. Let $\left\{\tilde{D}_{j}\right\}$ be defined as in Lemma

20. It is known (cite something here) that $\tilde{D}_{[\alpha \ell\rceil}$ in (24) satisfies $\lim _{\ell \rightarrow \infty} \tilde{D}_{\lceil\alpha \ell\rceil} \geq \ell h^{-1}(\alpha)$, where $h(\cdot)$ is the binary entropy function. Therefore, there exists an $\ell_{0}(\alpha)<\infty$ such that for all $\ell \geq \ell_{0}(\alpha)$ we have $D_{\lceil\alpha \ell\rceil} \geq \frac{1}{2} \ell h^{-1}(\alpha)$. Hence, for $\left\{\ell \geq \ell_{0}(\alpha)\right.$ we can write

$$
\mathrm{E}_{\ell} \geq \frac{1}{\ell} \sum_{i=\lceil\alpha \ell\rceil}^{\ell} \log _{\ell} \tilde{D}_{i}
$$




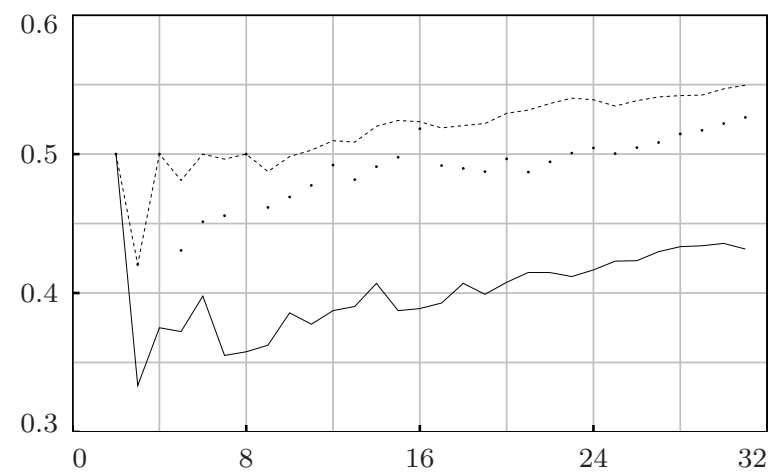

Fig. 3. The solid curve shows the lower bound on $\mathrm{E}_{\ell}$ as described by Lemma 20. The dashed curve corresponds to the upper bound on $\mathrm{E}_{\ell}$ according to Lemma 26. The points show the performance of the best matrices obtained by the procedure described in Section VI.

$$
\begin{aligned}
& \geq \frac{1}{\ell}(1-\alpha) \ell \log _{\ell} \tilde{D}_{\lceil\alpha \ell\rceil} \\
& \geq \frac{1}{\ell}(1-\alpha) \ell \log _{\ell} \frac{\ell h^{-1}(\alpha)}{2} \\
& =1-\alpha+(1-\alpha) \log _{\ell} \frac{h^{-1}(\alpha)}{2},
\end{aligned}
$$

where the first inequality follows from Lemma 20, and the second inequality follows from the fact that $\tilde{D}_{i} \leq \tilde{D}_{i+1}$ for all $i$. Therefore we obtain

$$
\liminf _{\ell \rightarrow \infty} \mathrm{E}_{\ell} \geq 1-\alpha \quad \forall \alpha \in\left(0, \frac{1}{2}\right) .
$$

Also, since $\tilde{D}_{i} \leq \ell$ for all $i$, we have $\mathrm{E}_{\ell} \leq 1$ for all $\ell$. Hence,

$$
\limsup _{\ell \rightarrow \infty} \mathrm{E}_{\ell} \leq 1
$$

Combining (25) and (26) concludes the proof.

\section{B. Upper Bound}

Corollary 19 says that for any $\ell$, there exists a matrix with $D_{1} \leq \cdots \leq D_{\ell}$ that achieves the exponent $\mathrm{E}_{\ell}$. Therefore, to obtain upper bounds on $\mathrm{E}_{\ell}$, it suffices to bound the exponent achievable by this restricted class of matrices. The partial distances of these matrices can be bounded easily as shown in the following lemma.

Lemma 22 (Upper Bound on Exponent): Let $d(n, k)$ denote the largest possible minimum distance of a binary code of length $n$ and dimension $k$. Then,

$$
\mathrm{E}_{\ell} \leq \frac{1}{\ell} \sum_{i=1}^{\ell} \log _{\ell} d(\ell, \ell-i+1) .
$$

Proof: Let $G$ be an $\ell \times \ell$ matrix with partial distances $\left\{D_{i}\right\}_{i=1}^{\ell}$ such that $\mathrm{E}(G)=\mathrm{E}_{\ell}$. Corollary 19 lets us assume without loss of generality that $D_{i} \leq D_{i+1}$ for all $i$. We therefore obtain

$$
D_{i}=\min _{j \geq i} D_{j}=\operatorname{dmin}\left(\left\langle g_{i}, \ldots, g_{\ell}\right\rangle\right) \leq d(\ell, \ell-i+1),
$$

where the second equality follows from Corollary 17 .

Lemma 22 allows us to use existing bounds on the minimum distances of binary codes to bound $\mathrm{E}_{\ell}$ :
Example 23 (Sphere Packing Bound): Applying the sphere packing bound for $d(\ell, \ell-i+1)$ in Lemma 22, we get

$$
\mathrm{E}_{\ell} \leq \frac{1}{\ell} \sum_{i=1}^{\ell} \log _{\ell} \tilde{D}_{i}
$$

where

$$
\tilde{D}_{i}=\max \left\{D: \sum_{j=0}^{\left\lfloor\frac{D-1}{2}\right\rfloor}\left(\begin{array}{l}
\ell \\
j
\end{array}\right) \leq 2^{i-1}\right\} .
$$

Note that for small values of $n$ for which $d(n, k)$ is known for all $k \leq n$, the bound in Lemma 22 can be evaluated exactly.

\section{Improved Upper Bound}

Bounds given in Section V-B relate the partial distances $\left\{D_{i}\right\}$ to minimum distances of linear codes, but are loose since they do not exploit the dependence among the $\left\{D_{i}\right\}$. In order to improve the upper bound we use the following parametrization: Consider an $\ell \times \ell$ matrix $G=\left[g_{1}^{T}, \ldots, g_{\ell}^{T}\right]^{T}$. Let

$$
\begin{aligned}
& T_{i}=\left\{k: g_{i k}=1, g_{j k}=0 \text { for all } j>i\right\} \\
& S_{i}=\left\{k: \exists j>i \text { s.t. } g_{j k}=1\right\},
\end{aligned}
$$

and let $t_{i}=\left|T_{i}\right|$.

Example 24: For the matrix

$$
F=\left[\begin{array}{llll}
0 & 0 & 0 & 1 \\
0 & 1 & 1 & 0 \\
1 & 1 & 0 & 0 \\
1 & 0 & 0 & 0
\end{array}\right]
$$

$T_{2}=\{3\}$ and $S_{2}=\{1,2\}$.

Note that $T_{i}$ are disjoint and $S_{i}=\cup_{j=i+1}^{\ell} T_{j}$. Therefore, $\left|S_{i}\right|=$ $\sum_{j=i+1}^{\ell} t_{i}$. Denoting the restriction of $g_{j}$ to the indices in $S_{i}$ by $g_{j S_{i}}$, we have

$$
D_{i}=t_{i}+s_{i}
$$

where $s_{i} \triangleq d_{H}\left(g_{i S_{i}},\left\langle g_{(i+1) S_{i}}, \ldots, g_{\ell S_{i}}\right\rangle\right)$. By a similar reasoning as in the proof of Lemma 18, it can be shown that there exists a matrix $G$ with

$$
s_{i} \leq d_{H}\left(g_{j S_{i}},\left\langle g_{(j+1) S_{i}}, \ldots, g_{\ell S_{i}}\right\rangle\right) \quad \forall i<j
$$

and

$$
\mathrm{E}(G)=\mathrm{E}_{\ell} .
$$

Therefore, for such a matrix $G$, we have (cf. proof of Lemma 22)

$$
s_{i} \leq d\left(\left|S_{i}\right|, \ell-i+1\right) .
$$

Using the structure of the set $S_{i}$, we can bound $s_{i}$ further:

Lemma 25 (Bound on Sub-distances): $s_{i} \leq\left\lfloor\frac{\left|S_{i}\right|}{2}\right\rfloor$.

Proof: We will find a linear combination of $\left\{g_{(i+1) S_{i}}, \ldots, g_{\ell S_{i}}\right\}$ whose Hamming distance to $g_{i S_{i}}$ is at most $\left\lfloor\frac{\left|S_{i}\right|}{2}\right\rfloor$. To this end define $w=\sum_{j=i+1}^{\ell} \alpha_{j} g_{j S_{i}}$, where $\alpha_{j} \in\{0,1\}$. Also define $w_{k}=\sum_{j=i+1}^{k} \alpha_{j} g_{j S_{i}}$. Noting that the sets $T_{j}$ s are disjoint with $\cup_{j=i+1}^{\ell} T_{j}=S_{i}$, we have $d_{H}\left(g_{i S_{i}}, w\right)=\sum_{j=i+1}^{\ell} d_{H}\left(g_{i T_{j}}, w_{T_{j}}\right)$. 
We now claim that choosing the $\alpha_{j} \mathrm{~s}$ in the order $\alpha_{i+1}, \ldots, \alpha_{\ell}$ by

$$
\operatorname{argmin}_{\alpha_{j} \in\{0,1\}} d_{H}\left(g_{i T_{j}}, w_{j-1 T_{j}}+\alpha_{j} g_{j T_{j}}\right),
$$

we obtain $d_{H}\left(g_{i S_{i}}, w\right) \leq\left\lfloor\frac{\left|S_{i}\right|}{2}\right\rfloor$. To see this, note that by definition of the sets $T_{j}$ we have $w_{T_{j}}=w_{j T_{j}}$. Also observe that by the rule (30) for choosing $\alpha_{j}$, we have $d_{H}\left(g_{i T_{j}}, w_{j T_{j}}\right) \leq\left\lfloor\frac{\left.\mid T_{j}\right\rfloor}{2}\right\rfloor$. Thus,

$$
\begin{aligned}
d_{H}\left(g_{i S_{i}}, w\right) & =\sum_{j=i+1}^{\ell} d_{H}\left(g_{i T_{j}}, w_{T_{j}}\right) \\
& =\sum_{j=i+1}^{\ell} d_{H}\left(g_{i T_{j}}, w_{j T_{j}}\right) \\
& \leq \sum_{j=i+1}^{\ell}\left\lfloor\frac{\left|T_{j}\right|}{2}\right\rfloor \leq\left\lfloor\frac{\left|S_{i}\right|}{2}\right\rfloor .
\end{aligned}
$$

Combining (28), (29) and Lemma 25, and noting that the invertibility of $G$ implies $\sum t_{i}=\ell$, we obtain the following:

Lemma 26 (Improved Upper Bound):

$$
\mathrm{E}_{\ell} \leq \max _{\sum_{i=1}^{\ell} t_{i}=\ell} \frac{1}{\ell} \sum_{i=1}^{\ell} \log _{\ell}\left(t_{i}+s_{i}\right)
$$

where

$$
s_{i}=\min \left\{\left\lfloor\frac{1}{2} \sum_{j=i+1}^{\ell} t_{j}\right\rfloor, d\left(\sum_{j=i+1}^{\ell} t_{j}, \ell-i+1\right)\right\} .
$$

The bound given in the above lemma is plotted in Figure 3. It is seen that no matrix with exponent greater than $\frac{1}{2}$ can be found for $\ell \leq 10$.

In addition to providing an upper bound to $\mathrm{E}_{\ell}$, Lemma 26 narrows down the search for matrices which achieve $E_{\ell}$. In particular, it enables us to list all sets of possible partial distances with exponents greater than $\frac{1}{2}$. For $11 \leq \ell \leq 14$, an exhaustive search for matrices with a "good" set of partial distances bounded by Lemma 26 (of which there are 285) shows that no matrix with exponent greater than $\frac{1}{2}$ exists.

\section{Vi. Construction Using BCH Codes}

We will now show how to construct a matrix $G$ of dimension $\ell=16$ with exponent exceeding $\frac{1}{2}$. In fact, we will show how to construct the best such matrix. More generally, we will show how $\mathrm{BCH}$ codes give rise to "good matrices." Our construction of $G$ consists of taking an $\ell \times \ell$ binary matrix whose $k$ last rows form a generator matrix of a $k$-dimensional $\mathrm{BCH}$ code. The partial distance $D_{k}$ is then at least as large as the minimum distance of this $k$-dimensional code.

To describe the partial distances explicitly we make use of the spectral view of $\mathrm{BCH}$ codes as sub-field sub-codes of Reed-Solomon codes as described in [4]. We restrict our discussion to BCH codes of length $\ell=2^{m}-1, m \in \mathbb{N}$.

Fix $m \in \mathbb{N}$. Partition the set of integers $\left\{0,1, \ldots, 2^{m}-2\right\}$ into a set $\mathcal{C}$ of chords,

$$
\mathcal{C}=\cup_{i=0}^{2^{m}-2}\left\{2^{k} i \quad \bmod \left(2^{m}-1\right): k \in \mathbb{N}\right\} .
$$

Example 27 (Chords for $m=5$ ): For $m=5$ the list of chords is given by

$$
\begin{aligned}
\mathcal{C}=\{ & \{0\},\{1,2,4,8,16\},\{3,6,12,17,24\}, \\
& \{5,9,10,18,20\},\{7,14,19,25,28\}, \\
& \{11,13,21,22,26\},\{15,23,27,29,30\}\} .
\end{aligned}
$$

Let $C$ denote the number of chords and assume that the chords are ordered according to their smallest element as in Example 27. Let $\mu(i)$ denote the minimal element of chord $i, 1 \leq i \leq C$ and let $l(i)$ denote the number of elements in chord $i$. Note that by this convention $\mu(i)$ is increasing. It is well known that $1 \leq l(i) \leq m$ and that $l(i)$ must divide $m$.

Example 28 (Chords for $m=5$ ): In Example 27 we have $C=7, l(1)=1, l(2)=\cdots=l(7)=5=m, \mu(1)=0$, $\mu(2)=1, \mu(3)=3, \mu(4)=5, \mu(5)=7, \mu(6)=11, \mu(7)=$ 15 .

Consider a $\mathrm{BCH}$ code of length $\ell$ and dimension $\sum_{j=k}^{C} l(j)$ for some $k \in\{1, \ldots, C\}$. It is well-known that this code has minimum distance at least $\mu(k)+1$. Further, the generator matrix of this code is obtained by concatenating the generator matrices of two $\mathrm{BCH}$ codes of respective dimensions $\sum_{j=k+1}^{C} l(j)$ and $l(k)$. This being true for all $k \in\{1, \ldots, C\}$, it is easy to see that the generator matrix of the $\ell$ dimensional (i.e., rate 1 ) $\mathrm{BCH}$ code, which will be the basis of our construction, has the property that its last $\sum_{j=k}^{C} l(j)$ rows form the generator matrix of a $\mathrm{BCH}$ code with minimum distance at least $\mu(k)+1$. This translates to the following lower bound on partial distances $\left\{D_{i}\right\}$ : Clearly, $D_{i}$ is least as large as the minimum distance of the code generated by the last $\ell-i+1$ rows of the matrix. Therefore, if $\sum_{j=k+1}^{C} l(j) \leq \ell-i+1 \leq \sum_{j=k}^{C} l(j)$, then

$$
D_{i} \geq \mu(k)+1 \text {. }
$$

The exponent $E$ associated with these partial design distances can then be bounded as

$$
\mathrm{E} \geq \frac{1}{2^{m}-1} \sum_{i=1}^{C} l(i) \log _{2^{m}-1}(\mu(i)+1) .
$$

Example 29 (BCH Construction for $\ell=31)$ : From the list of chords computed in Example 27 we obtain

$$
\mathrm{E} \geq \frac{5}{31} \log _{31}(2 \cdot 4 \cdot 6 \cdot 8 \cdot 12 \cdot 16) \approx 0.526433 .
$$

An explicit check of the partial distances reveals that the above inequality is in fact an equality.

For large $m$, the bound in (31) is not convenient to work with. The asymptotic behavior of the exponent is however easy to assess by considering the following bound. Note that no $\mu(i)$ (except for $i=1$ ) can be an even number since otherwise $\mu(i) / 2$, being an integer, would be contained in chord $i$, a contradiction. It follows that for the smallest exponent all chords (except chord 1) must be of length $m$ and that $\mu(i)=2 i+1$. This gives rise to the bound

$$
\begin{aligned}
\mathrm{E} \geq & \frac{1}{\left(2^{m}-1\right) \log \left(2^{m}-1\right)} \\
& \cdot\left(\sum_{k=1}^{a} m \log (2 k)+\left(2^{m}-2-a m\right) \log (2 a+2)\right),
\end{aligned}
$$


where $a=\left\lfloor\frac{2^{m}-2}{m}\right\rfloor$. It is easy to see that as $m \rightarrow \infty$ the above exponent tends to 1 , the best exponent one can hope for (cf. Lemma 21). We have also seen in Example 29 that for $m=5$ we achieve an exponent strictly above $\frac{1}{2}$.

Binary $\mathrm{BCH}$ codes exist for lengths of the form $2^{m}-1$. To construct matrices of other lengths, we use shortening, a standard method to construct good codes of smaller lengths from an existing code, which we recall here: Given a code $\mathrm{C}$, fix a symbol, say the first one, and divide the codewords into two sets of equal size depending on whether the first symbol is a 1 or a 0 . Choose the set having zero in the first symbol and delete this symbol. The resulting codewords form a linear code with both the length and dimension decreased by one. The minimum distance of the resulting code is at least as large as the initial distance. The generator matrix of the resulting code can be obtained from the original generator matrix by removing a generator vector having a one in the first symbol, adding this vector to all the remaining vectors starting with a one and removing the first column.

Now consider an $\ell \times \ell$ matrix $G_{\ell}$. Find the column $j$ with the longest run of zeros at the bottom, and let $i$ be the last row with a 1 in this column. Then add the $i$ th row to all the rows with a 1 in the $j$ th column. Finally, remove the $i$ th row and the $j$ th column to obtain an $(\ell-1) \times(\ell-1)$ matrix $G_{\ell-1}$. The matrix $G_{\ell-1}$ satisfies the following property.

Lemma 30 (Partial Distances after Shortening): Let the partial distances of $G_{\ell}$ be given by $\left\{D_{1} \leq \cdots \leq D_{\ell}\right\}$. Let $G_{\ell-1}$ be the resulting matrix obtained by applying the above shortening procedure with the $i$ th row and the $j$ th column. Let the partial distances of $G_{\ell-1}$ be $\left\{D_{1}^{\prime}, \ldots, D_{\ell-1}^{\prime}\right\}$. We have

$$
\begin{aligned}
& D_{k}^{\prime} \geq D_{k}, \quad 1 \leq k \leq i-1 \\
& D_{k}^{\prime}=D_{k+1}, \quad i \leq k \leq \ell-1 .
\end{aligned}
$$

Proof: Let $G_{\ell}=\left[g_{1}^{T}, \ldots, g_{\ell}^{T}\right]^{T}$ and $G_{\ell-1}=$ $\left[g_{1}^{\prime T}, \ldots, g_{\ell-1}^{\prime}\right]^{T}$ For $i \leq k, g_{k}^{\prime}$ is obtained by removing the $j$ th column of $g_{k+1}$. Since all these rows have a zero in the $j$ th position their partial distances do not change, which in turn implies (34).

For $k \leq i$, note that the minimum distance of the code $\mathrm{C}^{\prime}=$ $\left\langle g_{k}^{\prime}, \ldots, g_{\ell-1}^{\prime}\right\rangle$ is obtained by shortening $\mathrm{C}=\left\langle g_{k}, \ldots, g_{\ell}\right\rangle$. Therefore, $D_{k}^{\prime} \geq \operatorname{dmin}\left(\mathrm{C}^{\prime}\right) \geq \operatorname{dmin}(\mathrm{C})=D_{k}$.

Example 31 (Shortening of Code): Consider the matrix

$$
\left[\begin{array}{lllll}
1 & 0 & 1 & 0 & 1 \\
0 & 0 & 1 & 0 & 1 \\
0 & 1 & 0 & 0 & 1 \\
0 & 0 & 0 & 1 & 1 \\
1 & 1 & 0 & 1 & 1
\end{array}\right]
$$

The partial distances of this matrix are $\{1,2,2,2,4\}$. According to our procedure, we pick the 3 rd column since it has a run of three zeros at the bottom (which is maximal). We then add the second row to the first row (since it also has a 1 in the third column). Finally, deleting column 3 and row 2 we obtain the matrix

$$
\left[\begin{array}{llll}
1 & 0 & 0 & 0 \\
0 & 1 & 0 & 1 \\
0 & 0 & 1 & 1 \\
1 & 1 & 1 & 1
\end{array}\right]
$$

The partial distances of this matrix are $\{1,2,2,4\}$.

Example 32 (Construction of Code with $\ell=16$ ): Starting with the $31 \times 31 \mathrm{BCH}$ matrix and repeatedly applying the above procedure results in the exponents listed in Table I.

\begin{tabular}{c|c|c|c|c|c|c|c}
$\ell$ & exponent & $\ell$ & exponent & $\ell$ & exponent & $\ell$ & exponent \\
\hline 31 & 0.52643 & 27 & 0.50836 & 23 & 0.50071 & 19 & 0.48742 \\
30 & 0.52205 & 26 & 0.50470 & 22 & 0.49445 & 18 & 0.48968 \\
29 & 0.51710 & 25 & 0.50040 & 21 & 0.48705 & 17 & 0.49175 \\
28 & 0.51457 & 24 & 0.50445 & 20 & 0.49659 & 16 & 0.51828
\end{tabular}

\section{TABLE I}

THE BEST EXPONENTS ACHIEVED BY SHORTENING THE BCH MATRIX OF LENGTH 31.

The $16 \times 16$ matrix having an exponent 0.51828 is

$\left[\begin{array}{llllllllllllllll}1 & 0 & 0 & 1 & 1 & 1 & 0 & 0 & 0 & 0 & 1 & 1 & 1 & 1 & 0 & 1 \\ 0 & 1 & 0 & 0 & 1 & 0 & 0 & 1 & 0 & 1 & 1 & 1 & 0 & 0 & 1 & 1 \\ 0 & 0 & 1 & 1 & 1 & 1 & 1 & 0 & 0 & 1 & 1 & 0 & 1 & 1 & 1 & 0 \\ 0 & 1 & 0 & 1 & 0 & 1 & 1 & 0 & 1 & 0 & 1 & 0 & 0 & 0 & 0 & 0 \\ 1 & 1 & 1 & 1 & 0 & 0 & 0 & 0 & 0 & 0 & 1 & 0 & 1 & 1 & 0 & 1 \\ 0 & 0 & 1 & 0 & 0 & 1 & 0 & 1 & 0 & 1 & 0 & 0 & 0 & 1 & 1 & 0 \\ 0 & 0 & 1 & 0 & 0 & 0 & 0 & 0 & 0 & 1 & 1 & 1 & 0 & 0 & 0 & 0 \\ 0 & 1 & 0 & 1 & 1 & 1 & 0 & 0 & 1 & 0 & 1 & 1 & 0 & 0 & 1 & 0 \\ 1 & 1 & 1 & 0 & 0 & 1 & 1 & 0 & 1 & 0 & 0 & 1 & 0 & 1 & 0 & 0 \\ 1 & 0 & 1 & 0 & 1 & 0 & 1 & 1 & 1 & 0 & 1 & 1 & 0 & 1 & 0 & 1 \\ 1 & 1 & 1 & 0 & 0 & 0 & 0 & 0 & 0 & 0 & 0 & 1 & 1 & 0 & 1 & 0 \\ 1 & 0 & 0 & 1 & 1 & 0 & 0 & 0 & 0 & 1 & 0 & 1 & 1 & 0 & 1 & 1 \\ 1 & 1 & 1 & 1 & 1 & 0 & 1 & 0 & 0 & 0 & 0 & 1 & 0 & 1 & 0 & 0 \\ 1 & 0 & 1 & 0 & 1 & 1 & 1 & 1 & 0 & 1 & 0 & 0 & 0 & 0 & 0 & 1 \\ 1 & 0 & 1 & 0 & 0 & 0 & 0 & 1 & 0 & 1 & 1 & 1 & 1 & 1 & 0 & 0 \\ 1 & 1 & 1 & 1 & 1 & 1 & 1 & 1 & 1 & 1 & 1 & 1 & 1 & 1 & 1 & 1\end{array}\right]$

The partial distances of this matrix are $\{16,8,8,8,8,6,6,4,4,4,4,2,2,2,2,1\}$. Using Lemma 26 we observe that for the $16 \times 16$ case there are only 11 other possible sets of partial distances which have a better exponent than the above matrix. An exhaustive search for matrices with such sets of partial distances confirms that no such matrix exists. Hence, the above matrix achieves the best possible exponent among all $16 \times 16$ matrices.

\section{ACKNOWLEDGMENT}

We would like to thank E. Telatar for helpful discussions and his suggestions for an improved exposition. This work was partially supported by the National Competence Center in Research on Mobile Information and Communication Systems (NCCR-MICS), a center supported by the Swiss National Science Foundation under grant number 5005-67322.

\section{APPENDIX}

In this section we prove the following lemma which is used in the proof of Lemma 6. 
Lemma 33 (Mutual Information of $W^{k}$ ): Let $W$ be a symmetric B-DMC and let $W^{k}$ denote the channel

$$
W^{k}\left(y_{1}^{k} \mid x\right)=\prod_{i=1}^{k} W\left(y_{i} \mid x\right) .
$$

If $I(W) \in(\delta, 1-\delta)$ for some $\delta>0$, then there exists an $\eta(\delta)>0$ such that $I\left(W^{k}\right)-I(W)>\eta(\delta)$.

The proof of Lemma 33 is in turn based on the following theorem.

Theorem 34 ([5], [6] Extremes of Information Combining): Let $W_{1}, \ldots, W_{k}$ be $k$ symmetric B-DMCs with capacities $I_{1}, \ldots, I_{k}$ respectively. Let $W^{(k)}$ denote the channel with transition probabilities

$$
W^{(k)}\left(y_{1}^{k} \mid x\right)=\prod_{i=1}^{k} W_{i}\left(y_{i} \mid x\right) .
$$

Also let $W_{\mathrm{BSC}}^{(k)}$ denote the channel with transition probabilities

$$
W_{\mathrm{BSC}}^{(k)}\left(y_{1}^{k} \mid x\right)=\prod_{i=1}^{k} W_{\mathrm{BSC}\left(\epsilon_{i}\right)}\left(y_{i} \mid x\right),
$$

where $\operatorname{BSC}\left(\epsilon_{i}\right)$ denotes the binary symmetric channel (BSC) with crossover probability $\epsilon_{i} \in\left[0, \frac{1}{2}\right], \epsilon_{i} \triangleq h^{-1}\left(1-I_{i}\right)$, where $h$ denotes the binary entropy function. Then, $I\left(W^{(k)}\right) \geq$ $I\left(W_{\mathrm{BSC}}^{(k)}\right)$.

Remark 35: Consider the transmission of a single bit $X$ using $k$ independent symmetric B-DMCs $W_{1}, \ldots, W_{k}$ with capacities $I_{1}, \ldots, I_{k}$. Theorem 34 states that over the class of all symmetric channels with given mutual informations, the mutual information between the input and the output vector is minimized when each of the individual channels is a BSC.

Proof of Lemma 33: Let $\epsilon \in\left[0, \frac{1}{2}\right]$ be the crossover probability of a BSC with capacity $I(W)$, i.e., $\epsilon=h^{-1}(1-I(W))$. Note that for $k \geq 2$,

$$
I\left(W^{k}\right) \geq I\left(W^{2}\right) \geq I(W) .
$$

By Theorem 34, we have $I\left(W^{2}\right) \geq I\left(W_{\mathrm{BSC}(\epsilon)}^{2}\right)$. A simple computation shows that

$$
I\left(W_{\mathrm{BSC}(\epsilon)}^{2}\right)=1+h(2 \epsilon \bar{\epsilon})-2 h(\epsilon) .
$$

We can then write

$$
\begin{aligned}
I\left(W^{k}\right)-I(W) & \geq I\left(W_{\mathrm{BSC}(\epsilon)}^{2}\right)-I(W) \\
& =I\left(W_{\mathrm{BSC}(\epsilon)}^{2}\right)-I\left(W_{\mathrm{BSC}(\epsilon)}\right) \\
& =h(2 \epsilon \bar{\epsilon})-h(\epsilon) .
\end{aligned}
$$

Note that $I(W) \in(\delta, 1-\delta)$ implies $\epsilon \in\left(\phi(\delta), \frac{1}{2}-\phi(\delta)\right)$ where $\phi(\delta)>0$, which in turn implies $h(2 \epsilon \bar{\epsilon})-h(\epsilon)>\eta(\delta)$ for some $\eta(\delta)>0$.

Lemma 36: Consider a symmetric B-DMC $W$. Let $P_{e}(W)$ denote the bit error probability of uncoded transmission under MAP decoding. Then,

$$
P_{e}(W) \geq \frac{1}{2}\left(1-\sqrt{1-Z(W)^{2}}\right) .
$$

Proof: One can check that the inequality is satisfied with equality for BSC. It is also known that any symmetric BDMC $W$ is equivalent to a convex combination of several, say
$K$, BSCs where the receiver has knowledge of the particular BSC being used. Let $\left\{\epsilon_{i}\right\}_{i=1}^{K}$ and $\left\{Z_{i}\right\}_{i=1}^{K}$ denote the bit error probabilities and the Bhattacharyya parameter of the constituent BSCs. Then, $P_{e}(W)$ and $Z(W)$ are given by

$$
P_{e}(W)=\sum_{i=1}^{K} \alpha_{i} \epsilon_{i}, \quad Z(W)=\sum_{i=1}^{K} \alpha_{i} Z_{i}
$$

for some $\alpha_{i}>0$, with $\sum_{i=1}^{K} \alpha_{i}=1$. Therefore,

$$
\begin{aligned}
P_{e}(W) & =\sum_{i=1}^{K} \alpha_{i} \frac{1}{2}\left(1-\sqrt{1-Z_{i}^{2}}\right) \\
& \geq \frac{1}{2}\left(1-\sqrt{1-\left(\sum_{i=1}^{K} \alpha_{i} Z_{i}\right)^{2}}\right) \\
& =\frac{1}{2}\left(1-\sqrt{1-Z(W)^{2}}\right),
\end{aligned}
$$

where the inequality follows from the convexity of the function $x \rightarrow 1-\sqrt{1-x^{2}}$ for $x \in(0,1)$.

\section{REFERENCES}

[1] E. Arıkan, "Channel polarization: A method for constructing capacityachieving codes for symmetric binary-input memoryless channels," submitted to IEEE Trans. Inform. Theory, 2008.

[2] E. Arikan and E. Telatar, "On the rate of channel polarization," July 2008, available from "http://arxiv.org/pdf/0807.3917".

[3] J.A. Bondy and U.S.R. Murty, Graph Theory. Springer, 2008.

[4] R. E. Blahut, Theory and Practice of Error Control Codes. AddisonWesley, 1983.

[5] I. Sutskover, S. Shamai, and J. Ziv, "Extremes of information combining," IEEE Trans. Inform. Theory, vol. 51, no. 4, pp. 1313 - 1325, Apr. 2005.

[6] I. Land, S. Huettinger, P. A. Hoeher, and J. B. Huber, "Bounds on information combining," IEEE Transactions on Information Theory, vol. 51, no. 2, pp. 612-619, 2005. 